\title{
The dependences of electroluminescent characteristics of ZnS:Mn thin films upon their device parameters
}

\author{
Hiroshi Sasakura, Hiroshi Kobayashi, Shosaku Tanaka, Juro Mita, ${ }^{\text {") }}$ \\ Toshihiko Tanaka, ${ }^{\text {b) }}$ and Hirofumi Nakayama c) \\ Department of Electronics, Tottori University, Koyama, Tottori 680, Japan
}

(Received 16.April 1981; accepted for publication 7 July 1981)

The dependences of brightness, emission efficiency $\eta$, average electric field $E_{A}$, conduction current $J_{A}$, and emission lifetime $\tau$ upon the device parameters such as film thickness, substrate temperature during evaporation, and $\mathrm{Mn}$ concentration have been systematically investigated in $\mathrm{ZnS}: \mathrm{Mn}$ thin-film electroluminescent devices. The value of $\eta$ increases rapidly with film thicknesses below $3000 \AA \AA$ but $E_{A}$ decreases slowly. These results can be explained by the increase of the crystallinity of the ZnS:Mn films. The value of $\eta$ increases with the Mn concentration and reaches its maximum at about $0.45 \mathrm{wt} \%$. At above this $\mathrm{Mn}$ concentration, $\eta$ and $\tau$ decrease rapidly, $E_{A}$ increases, and $\dot{J}_{A}$ decreases slowly. These results may be attributed to a decrease of hot electron energy and/or an increase of the nonradiative transition probability of the excited Mn centers. The brightness-voltage $(B-V)$ hysteresis characteristic is observed in this $\mathbf{M n}$ concentration region. This memory effect is also discussed.

PACS numbers: $78.60 . F i$ 85.60.Jb

\section{INTRODUCTION}

Electroluminescent (EL) devices are available for completely solid displays. A large-area display can also be easily realized by use of these devices. Because of their high brightness and long life, ${ }^{1,2}$ many studies have recently been made of thin-film EL devices consisting of a ZnS:Mn layer sandwiched between two insulating layers. ${ }^{\mathrm{i}-11}$ However, our information about the mechanism of electroluminescence in devices of this type is not yet sufficient, even though several studies on this mechanism have been already carried out. ${ }^{4,5,8,12}$ In order to elucidate the mechanism of electroluminescence, it is necessary to make careful studies on the dependences of various $E L$ characteristics on various device parameters. Among the various EL characteristics of the devices, brightness and EL emission efficiency are the most important, but conduction current, electric field of the ZnS:Mn active layer, and decay time of EL emission are also important. Several studies have been reported on the dependences of individual EL characteristics on the device parameters, ${ }^{4,8,10}$ for example, the dependence of brightness on $\mathrm{Mn}$ concentration. ${ }^{8,10}$ However, no systematic study has been carried out on the dependences of the above mentioned EL characteristics upon various device parameters.

We have systematically investigated the dependences of these EL characteristics of the devices upon the device parameters, such as film thickness of the ZnS:Mn active layer, substrate temperature during evaporation, and $\mathrm{Mn}$ concentration of the active layer. These quantities have a remarkable affect on the EL characteristics, especially the brightness and the EL emission efficiency. From the experimental

\footnotetext{
"Present address: Oki Electric Industries, Co., Ltd., Toranomon, Minatoku, Tokyo 105, Japan.

bresent address: Tottori Sanyo Electric Co, Ltd., Tachikawa, Tottori 680, Japan.

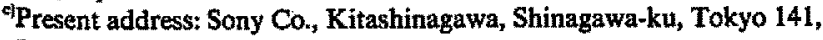
Japan.
}

results obtained, the mechanism of electroluminescence of thin film EL devices having a sandwich structure is discussed, and then the relationships between the EL characteristics and the device parameters are considered. In addition, the dependences of memory characteristics on the device parameters have been measured, and the memory mechanism is also discussed.

\section{EXPERIMENTAL METHOD}

The EL device structure used in the present study is shown in Fig. 1. The devices were prepared by sequential depositions of $\mathrm{Y}_{2} \mathrm{O}_{3}, \mathrm{ZnS}: \mathrm{Mn}, \mathrm{Y}_{2} \mathrm{O}_{3}$ again, and finally $\mathrm{Al}$ onto an indium-tin-oxide (ITO) coated glass plate. The ZnS:Mn film was used as an EL emission layer, and the $\mathrm{Y}_{2} \mathrm{O}_{3}$ films were used as insulating layers. All these depositions were carried out by electron beam evaporation. A mixture of $\mathrm{ZnS}$ powder and $\mathrm{Mn}$ metal was used as the evaporation source of the $\mathrm{ZnS}: \mathrm{Mn}$ film. The Mn concentration strongly affects the brightness and the memory characteristics of the device prepared. In our experiments, the Mn concentration was varied from 0.03 to $10 \mathrm{wt} \%$. However, the constant $\mathrm{Mn}$ concentration of $0.45 \mathrm{wt} \%$ was chosen unless otherwise mentioned, because this concentration exhibits the maxi-

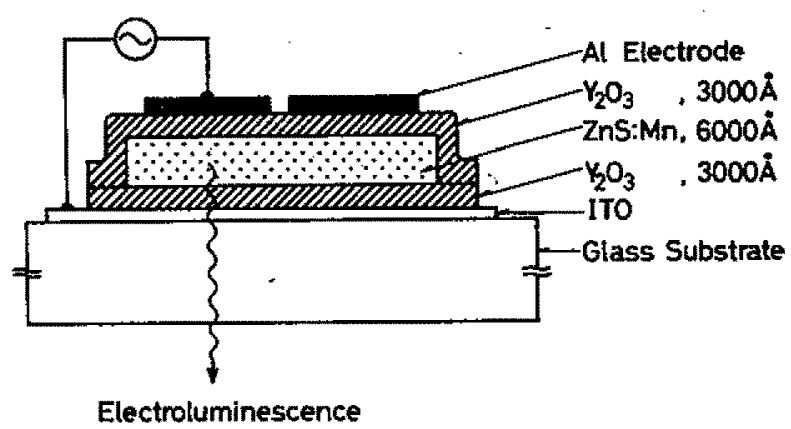

FIG. 1. Typical structure of a ZnS:Mn EL device. 
mum efficiency as well as the strongest brightness. The thickness of the ZnS:Mn film and the substrate temperature during evaporation were varied from 700 to $8000 \AA$ and 50 to $160^{\circ} \mathrm{C}$, respectively. The substrate temperature during evaporation of $\mathrm{Y}_{2} \mathrm{O}_{3}$ was $50^{\circ} \mathrm{C}$. The thickness of each $\mathrm{Y}_{2} \mathrm{O}_{3}$ insulating film was $3000 \AA$, which is the minimum value necessary to obtain stable operation of the device.

The EL characteristics were measured under the condition of driving the devices with $1 \mathrm{KHz}$ sinusoidal excitation unless otherwise mentioned. When the ac applied voltage is lower than the threshold voltage for EL emission, a large displacement current, and a small conduction current which is due to the losses of the electrodes and the insulating layers, flow in the ac EL device. When the applied voltage exceeds the threshold voltage, the conduction current, sometimes called the dissipative current, begins to increase rapidly. In the present study, the instantaneous change and the time average of the conduction current were measured by a bridge circuit composed of the EL device and a capacitor having a capacitance equal to that of the EL device. The relative EL emission efficiency $\eta$ was calculated as the ratio of the relative emission intensity to the real power loss in the EL device.

\section{RESULTS AND DISCUSSION}

\section{A. Crystallinity of ZnS:Mn EL emission layers}

In general, the crystallinity and orientation of evaporated films strongly depend on evaporation conditions such as substrate temperatures and underlying materials. In order to study the crystallinity of the ZnS:Mn EL layers, glass-ITO$\mathrm{Y}_{2} \mathrm{O}_{3}-\mathrm{ZnS}: \mathrm{Mn}$ multilayer films were prepared as samples for $\mathrm{x}$-ray diffraction measurements in our work. The $\mathrm{x}$-ray diffraction patterns of these samples are shown in Fig. 2. Substrate temperatures during evaporations of the $\mathrm{ZnS}: \mathrm{Mn}$ films were 120 and $50^{\circ} \mathrm{C}$, and the thicknesses of both these films were $6000 \AA$. As shown in Fig. 2(a), the $\mathrm{ZnS}: \mathrm{Mn}$ thin film evaporated at a substrate temperature of $120^{\circ} \mathrm{C}$ shows

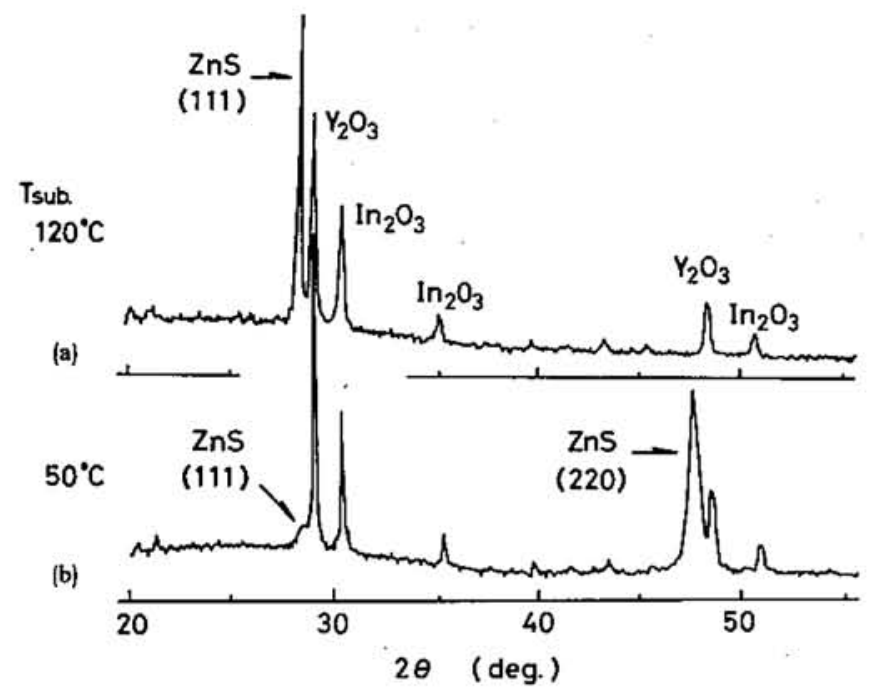

FIG. 2. X-ray diffraction patterns of the $\mathrm{ZnS}: \mathrm{Mn}$ films using a $\mathrm{Cu} \mathrm{K} \alpha$ line. Substrate temperatures during evaporation are 120 and $50^{\circ} \mathrm{C}$, as shown in (a) and (b), respectively.
TABLE I. The x-ray diffraction intensities $I_{\|_{11}^{*}}$ and $I_{220}^{*}$ of the $\mathrm{ZnS}: \mathrm{Mn}$ films and the EL maximum efficiency $\eta_{\max }$ (see Fig. 4) of the devices. The data have been obtained at several substrate temperatures during evaporation. The $I_{11}^{*}$ and $I_{220}^{*}$ are the intensities of the (111) and (220) lines per unit film thickness, respectively.

\begin{tabular}{rccc}
\hline \hline $\mathrm{T}_{\text {sub. }}\left({ }^{\circ} \mathrm{C}\right)$ & $I_{111}^{*}$ & $I_{\text {220 }}^{*}$ & $\eta_{\max }$ \\
\hline 50 & 0.4 & 9.3 & 0.28 \\
120 & 3.4 & $\cdots$ & 14.6 \\
160 & 9.0 & $\ldots$ & 18.3 \\
\hline
\end{tabular}

only the (111) line of the cubic structure of $\mathrm{ZnS}$. In other words, the $\mathrm{ZnS}: \mathrm{Mn}$ film strongly orientates its $\{111\}$ surface parallel to the substrate. All other lines observed in the diffraction pattern are due to underlying $\mathrm{Y}_{2} \mathrm{O}_{3}$ and $\mathrm{In}_{2} \mathrm{O}_{3}$ films. Similar results have also been obtained for the $\mathrm{ZnS}$ :Mn films evaporated at substrate temperatures from 80 to $160^{\circ} \mathrm{C}$. In our experiment, it was difficult to grow $\mathrm{ZnS}$ film at substrate temperatures above $200^{\circ} \mathrm{C}$. As shown in Fig. 2(b), we found that the strong (220) line and the weak (i11) line are observed for the $\mathrm{ZnS}: \mathrm{Mn}$ film evaporated at a substrate temperature of $50^{\circ} \mathrm{C}$. These results show that the orientation of $\mathrm{ZnS}: \mathrm{Mn}$ films strongly depends on the substrate temperature during evaporation. Recently the hexagonal structure of a $\mathrm{ZnS}$ film, which was prepared at a substrate temperature above $350^{\circ} \mathrm{C}$ by the use of the atomic-layer epitaxy method, has been reported. ${ }^{13}$

Table I shows the x-ray diffraction intensities per unit thicknesses $I_{11}^{*}, I_{220}^{*}$, and the relative EL maximum efficiencies $\eta_{\max }$ of the devices having these $\mathrm{ZnS}$ :Mn films. The value of $\eta_{\max }$ was determined from the dependence on applied voltage, as described in Sec. III B. The ZnS:Mn films were prepared at the substrate temperatures $T_{\text {sub }} 50,120$ and $160^{\circ} \mathrm{C}$. As shown in Table I, $I_{11}^{*}$ rapidly increases with the substrate temperature. Simultaneously, $\eta_{\max }$ also increases

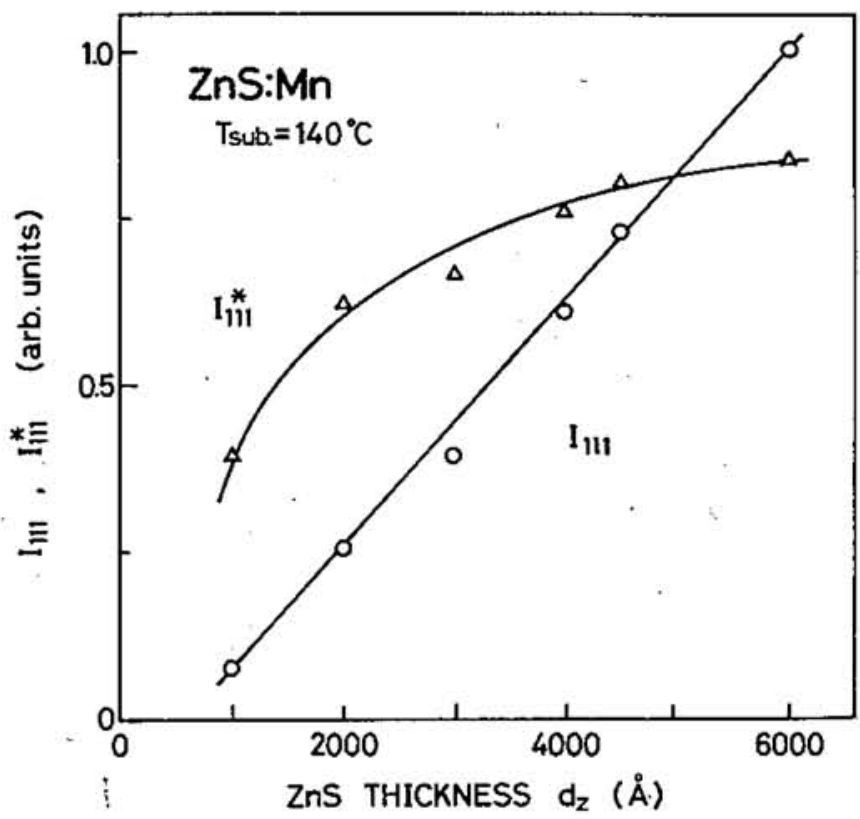

FIG. 3. Dependences of $I_{111}$ and $I_{711}^{*}$ on the thickness of the ZnS:Mn film, where $I_{111}$ is the intensity of the (111) line and $I_{11}^{*}$ is the intensity of the (111) line per unit thickness. 
with the substrate temperature. This result strongly suggests that $\eta_{\max }$ depends on the crystallinity of the ZnS:Mn films.

The dependence of the intensity of the (111) line $I_{111}$ upon the film thickness of the $\mathrm{ZnS}: \mathrm{Mn} d_{z}$ was measured in the case of the films prepared at the substrate temperature $T_{\text {sub }}$ of $140^{\circ} \mathrm{C}$. Only the (111) line is observed in this preparation condition, and the (220) line is not observed. The result is shown in Fig. 3. The intensity per unit thickness $I_{11}^{*}$ is also shown. The (111) line is hardly observed at film thicknesses below $1000 \AA$. The (111) line appears at about $1000 \AA$, and $I_{111}$ increases linearly with increasing film thickness. Therefore, $I_{111}^{*}$ rapidly increases in the neighborhood of a film thickness of $1000 \AA$, and tends to saturate gradually above about $4000 \AA$. The saturation of $I_{111}^{*}$ with increasing film thickness is explained as follows. In the neighborhood of the $\mathrm{ZnS} / \mathrm{Y}_{2} \mathrm{O}_{3}$ interface, the crystallinity of the $\mathrm{ZnS}: \mathrm{Mn}$ film is not good, because the lattice constant of $\mathrm{ZnS}(a=5.4060$ $\AA)$ is about $2 \%$ larger than that of $\mathrm{Y}_{2} \mathrm{O}_{3}(a / 2=5.30 \AA)$. When the ZnS:Mn film grows larger than $1000 \AA$, the crystallinity of the film becomes better, and the $\{111\}$ plane of $\mathrm{ZnS}$ begins to orientate parallel to the substrate. Therefore, $I_{111}^{*}$ increases with the film thickness, and it eventually saturates at film thicknesses above $4000 \AA$.

\section{B. The dependences of the brightness and the EL emission efficiency on the $\mathrm{ZnS}: \mathrm{Mn}$ film thickness}

The dependences of the brightness $B$ and the relative EL emission efficiency $\eta$ on the applied voltage are shown in Fig. 4, where the thickness of the $\mathrm{ZnS}$ :Mn film $d_{z}$ is chosen as a parameter. The Mn concentration was $0.45 \mathrm{wt} \%$ and the substrate temperature during evaporation was $140^{\circ} \mathrm{C}$. When the film thickness is $6900 \AA$, the brightness $B$ increases rapidly at an applied voltage of $140 \sim 170 \mathrm{~V}$, and it tends to saturate above $200 \mathrm{~V}$. The maximum brightness of about $1000 \mathrm{fL}$ is obtained at an applied voltage of $230 \mathrm{~V}$. However, the maximum efficiency $\eta_{\max }$ is obtained at about $170 \mathrm{~V}$. When the film thickness is $1400 \AA, \eta_{\max }$ is obtained at about $130 \mathrm{~V}$. When the film thickness is decreased to $700 \AA, \eta_{\max }$ is obtained at about $150 \mathrm{~V}$, which is slightly higher than that of the film thickness $1400 \AA$. In discussing the dependence of the brightness on the film thickness, we define the brightness $B_{A}$ at the voltage $V_{A}$, at which $\eta_{\max }$ is obtained (see Fig. 4). The electric field of the $\mathrm{ZnS}: \mathrm{Mn}$ layer is nearly clamped above $V_{A} A^{3,14,15}$ To discuss the film thickness and the Mn concentration dependences of the electric field, therefore, we use the electric field $E_{A}$ at $V_{A}$, which is considered to be a representative value of the electric field. Strictly speaking, the $\mathrm{ZnS}: \mathrm{Mn}$ film is not homogeneous as described below so that the electric field within it is not constant. However, for simplification, we may define $E_{A}$ as the average electric field in the film. We have measured the electric field $E_{A}$ by measuring the instantaneous conduction current, ${ }^{14}$ and also estimated $E_{A}$ from the average conduction current. The value of $E_{A}$ obtained with the above two methods coincided well with each other. The voltage $V_{A}$ decreases when the thickness of the $\mathrm{ZnS}: \mathrm{Mn}$ film is changed from 700 to $1400 \AA$. This result clearly shows that the electric field of the film decreases with the film thickness at least in the above-men-

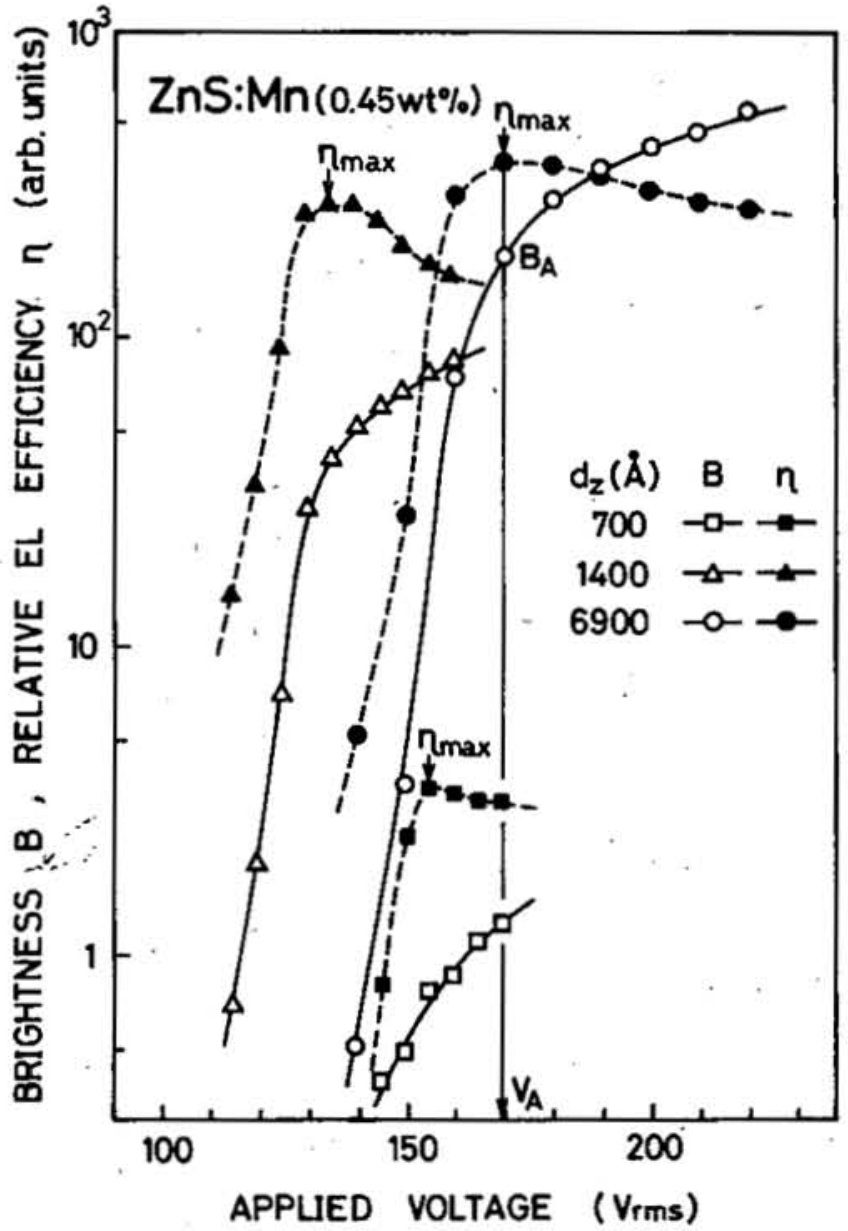

FIG. 4. Voltage dependences of brightness $B$ and relative EL emission efficiency $\eta$. The thickness of the $\mathrm{ZnS}: \mathrm{Mn}$ film $d_{z}$ is taken as a parameter. The $B_{A}$ and $V_{A}$ obtained at $\eta_{\max }$ are also shown in the case of $d_{z} 6900 \AA$.

tioned range of film thicknesses, although the brightness and the EL emission efficiency increase.

Figure 5 shows the dependences of $B_{A}, \eta_{\max }$, and $E_{A}$ on the thickness of the ZnS:Mn film $d_{z}$, together with $I_{11}^{*}$, which has already been shown in Fig. 3. The value of $E_{A}$ is about $3 \times 10^{6} \mathrm{~V} / \mathrm{cm}$ at a film thickness $d_{z}$ of $700 \AA$. As described above, $E_{A}$ decreases gradually with increasing $d_{z}$ and reaches about $1.5 \times 10^{6} \mathrm{~V} / \mathrm{cm}$ at $d_{z} 3000 \AA$. In any further increase of $d_{z}, E_{A}$ remains nearly constant. On the other hand, $B_{A}$ first increases rapidly with an increasing $d_{z}$, and it increases nearly proportionally to $d_{z}$ above $3000 \AA$. The maximum EL efficiency $\eta_{\max }$ increases rapidly from a filmthickness $d_{z}$ of $700 \AA$ and reaches its largest value at a $d_{z}$ of about $3000 \AA$. The increase of the $\eta_{\max }$ is more than $10^{2}$, times in this film thickness range. In any further increase of $d_{z}, \eta_{\max }$ keeps nearly constant. $I_{11}^{*}$ increases gradually with increasing $d_{z}$, and it stays at nearly a constant value above $3000 \AA$. The tendency of variation of $I_{11}^{*}$ with increases in $d_{z}$ is similar to that of $\eta_{\max }$. The increase of $I_{111}^{*}$ gives direct evidence that the crystallinity of the $\mathrm{ZnS}: \mathrm{Mn}$ film improves with increased film thickness. Therefore, it seems very likely that a close correlation exists between $\eta_{\max }$ and the crystallinity of the $\mathrm{ZnS}: \mathrm{Mn}$ film. It has been already reported that the low EL emission efficiency of $\mathrm{ZnS}: \mathrm{Mn}$ film is observed at film thicknesses below $2000 \AA$, and it has been suggested that 


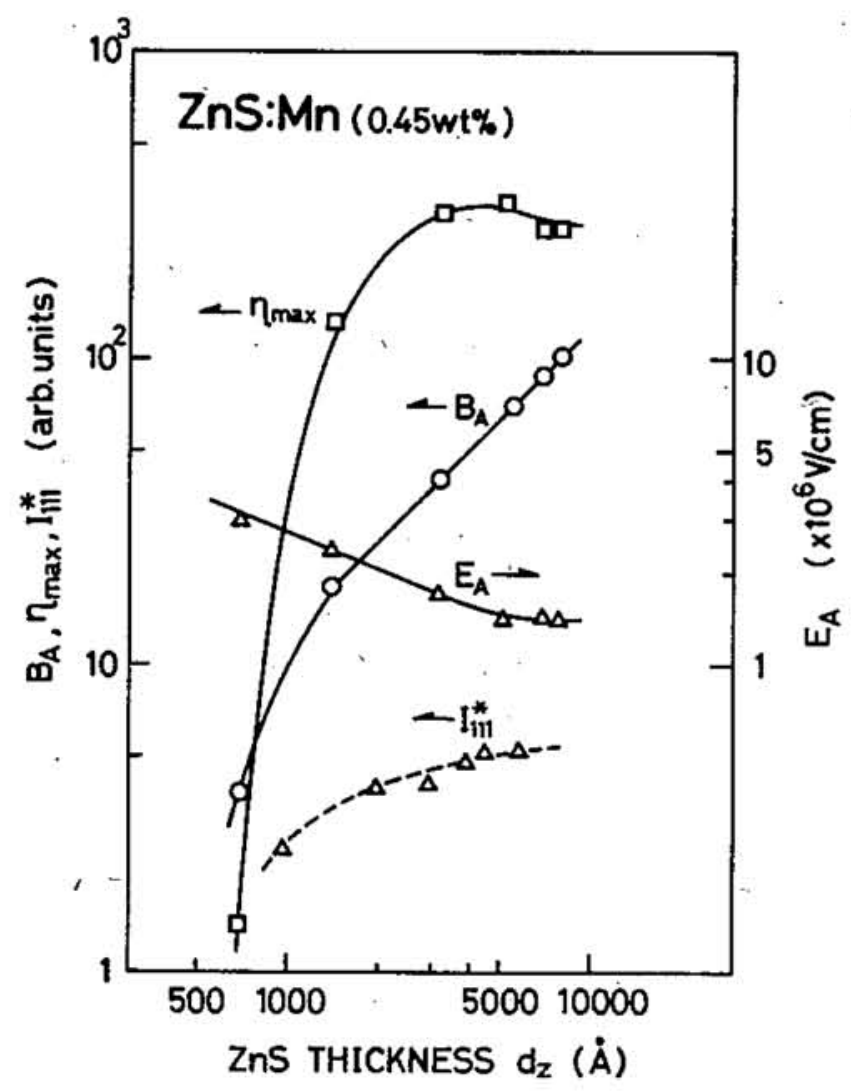

FIG. 5. Dependences of brightness $B_{A}$, the maximum EL emission efficiency $\eta_{\max }$, and the electric field of the $\mathrm{ZnS}: \mathrm{Mn}$ film $E_{A}$ upon the film thickness of the $\mathrm{ZnS}: \mathrm{Mn}$ active layer. The $B_{A}$ and $E_{A}$ are measured at the voltage $V_{A}$, at which $\eta_{\max }$ is obtained. The dependence of $I_{i 1}^{*}$ is also shown.

this low EL efficiency may be correlated to the crystal morphology of the ZnS:Mn film. ${ }^{12,16}$ Our results are well consistent with this suggestion.

The close correlation between $\eta_{\max }$ and the crystallinity of the $\mathrm{ZnS}: \mathrm{Mn}$ film can be explained as follows. As the excitation mechanism is due to impact excitation by hot electrons, ${ }^{5} \eta_{\max }$ depends on the energy distribution of the hot èlectrons. This distribution can be represented as a function of $e E_{A} \lambda,,^{14,17}$ where $e$ is a charge of an electron and $\lambda$ is the mean free path of hot electrons. From the analysis, it is clear that $\eta_{\max }$ increases rapidly when $e E_{A} \lambda$ increases slightly.

We first discuss the dependence of $E_{A}$ and $\lambda$ on the crystallinity, which in turn depends on the film thickness. In general, $\lambda$ is considered in homogeneous material. However, the $\mathrm{ZnS}: \mathrm{Mn}$ EL emission layer is an inhomogeneous layer, in which the crystallinity becomes better the greater the distance from the $\mathrm{ZnS} / \mathrm{Y}_{2} \mathrm{O}_{3}$ interface. It is well-known that $\lambda$ increases when the crystallinity of a solid improves. Therefore, $\lambda$ can be regarded as increasing with increasing distance from the $\mathrm{ZnS} / \mathrm{Y}_{2} \mathrm{O}_{3}$ interface in the $\mathrm{ZnS}: \mathrm{Mn}$ layer. As the average energy of hot electrons increases with increasing $\lambda$, it increases with increasing film thickness, so that the avalanche breakdown field $E_{A}$ (Ref. 15) is expected to become lower. This expectation agrees with the present experimental results concerning $E_{A}$.

We next discuss the maximum efficiency $\eta_{\max }$. As described above, $\eta_{\max }$ is also the function of $e E_{A} \lambda$ and increases rapidly when $e E_{A} \lambda$ increases even slightly. ${ }^{14} \mathrm{~W}$ ith an increasing film thickness, $\lambda$ increases and $E_{A}$ decreases. The observed decrease of $E_{A}$ is from $3 \times 10^{6}$ to $1.5 \times 10^{6} \mathrm{~V} / \mathrm{cm}$ by increasing the film thickness from 700 to $3000 \AA$. In other words, $E_{A}$ is reduced to about half its former value in this range of film thicknesses. However, if $\lambda$ becomes $3 \sim 5$ times larger at that time, $e E_{A} \lambda$ becomes about twice as large. From this, one can explain the observed increase of $\eta_{\max }$ of about $10^{2}$ times as being due to the increase of $e E_{A} \lambda$.

\section{Dependences of $B_{A}, \eta_{\max }, E_{A}, J_{A}$, and $\tau$ on the $M n$ concentration}

Figure 6 shows the dependences of $B_{A}, \eta_{\max }$, and $E_{A}$ on the Mn concentration of the $\mathrm{ZnS}: \mathrm{Mn} \mathrm{EL}$ active layer. The $\mathrm{Mn}$ concentration was varied from 0.03 to $10 \mathrm{wt} \%$. The ZnS:Mn film of thickness $6000 \AA$ was prepared at the substrate temperature $140^{\circ} \mathrm{C}$ during evaporation. The applied voltage dependence of the average conduction current $J$ has been measured, and it increases nearly proportionally to $\left(V-V_{\mathrm{th}}\right)$, where $V_{\mathrm{th}}$ is the threshold voltage for EL emission. Figure 6 also shows the concentration dependences of the conduction current $J_{A}$, defined at the voltage $V_{A}$, because it is a current under the electric field $E_{A}$ at which the avalanche breakdown begins to occur in the $\mathrm{ZnS}$ :Mn layer. This value seems to be useful in discussing conduction mechanisms of $\mathrm{ZnS}: \mathrm{Mn} \mathrm{EL}$ layers having various $\mathrm{Mn}$ con-

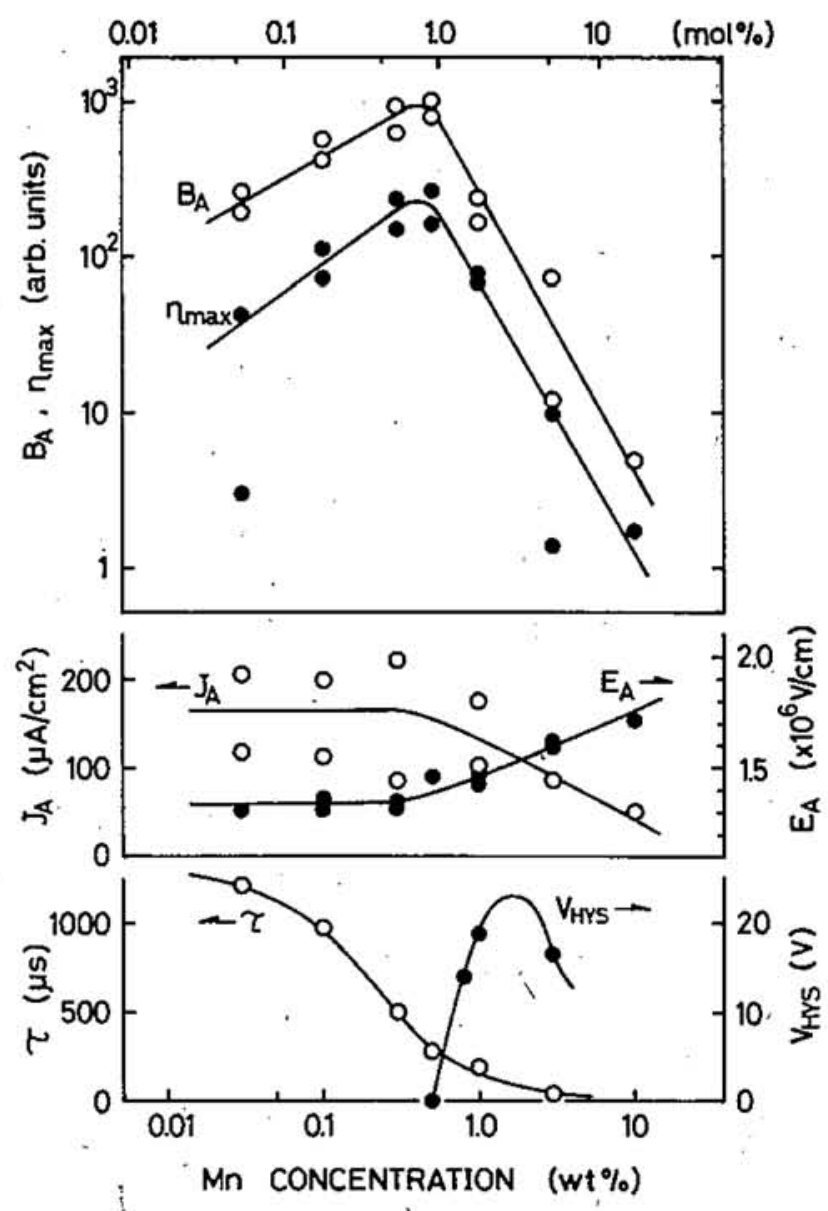

FIG. 6. Dependences of $B_{A}, \eta_{\max }, E_{A}, J_{A}$, and $\tau$ on the Mn concentration of the $\mathrm{ZnS}: \mathrm{Mn}$ active layer, where $J_{A}$ is the conduction current density of the active layer and $\tau$ is the decay time of EL emission. The dependence of the hysteresis width $V_{\text {HYs }}$ on the Mn concentration is also shown. 
centrations. The decay time of the Mn electroluminescence $\tau$, which is measured by applying the pulse voltage (rise time $<10 \mu$ s, pulse width $\sim 100 \mu$ s) to the device, is also shown in Fig. 6.

In the region of low $\mathrm{Mn}$ concentration, $B_{A}$ and $\eta_{\max }$ increase with increasing Mn concentration and reach maximum at an Mn concentration of $0.45 \mathrm{wt} \%$. Unlike $B_{A}$ and $\eta_{\max }, J_{A}$ and $E_{A}$ are nearly constant when the Mn concentration is below $0.5 \mathrm{wt} \%$, but $J_{A}$ begins to decrease and $E_{A}$ begins to increase as the Mn concentration becomes higher. Furthermore, the larger conduction current $J$ at a higher voltage than $V_{A}$ tends to decrease with Mn concentration as well as $J_{A}$. The brightness-voltage $(B-V)$ hysteresis characteristic, the so-called memory effect, ${ }^{2}$ is observed when the device has an Mn concentration of more than $0.5 \mathrm{wt} \%$. The hysteresis voltage width $V_{\text {HYS }}$ is also shown in Fig. 6. This memory effect is discussed in Sec. III D.

In this section, the dependences of $E_{A}$ and $J_{A}$ on the $\mathrm{Mn}$ concentration are first discussed. $E_{A}$ is considered to be determined by the avalanche breakdown process. ${ }^{15}$ In our experiment, $E_{A}$ increased with increasing Mn concentration. Similar behavior of a threshold electric field has also been observed recently. ${ }^{8}$ From our observation, it seems that the mean free path of electrons $\lambda$ may decrease in the high $\mathrm{Mn}$ concentration region, because the avalanche breakdown of the $\mathrm{ZnS}: \mathrm{Mn}$ layer having higher $\mathrm{Mn}$ concentration occurs in higher electric fields, as long as the value of $e E_{A} \lambda$ keeps to a certain value. The decrease of $\lambda$ is presumably due to the increase of the scattering probability of hot electrons by $\mathrm{Mn}$ centers and/or the decrease of the crystallinity in the

$\mathrm{ZnS}: \mathrm{Mn}$ layer. On the other hand, $J_{A}$ decreases with increasing Mn concentrations. We may point out that there are two possible causes for the decrease of $J_{A}$. One is a decrease of the electron density and the other is a decrease in the length of the mean free path. However, our knowledge of the conduction mechanism in $\mathrm{ZnS}: \mathrm{Mn}$ under high electric fields is still insufficient to enable us to draw a valid conclusion.

Next, the dependences of $B_{A}$ and $\eta_{\max }$ on the Mn concentration are discussed. In the low Mn concentration region below $0.3 \mathrm{wt} \%$, both $B_{A}$ and $\eta_{\max }$ increase with the $\mathrm{Mn}$ concentration. In this concentration region, $E_{A}$ and $J_{A}$ are nearly constant, that is to say, the energy distribution of hot electrons in the $\mathrm{ZnS}: \mathrm{Mn}$ layer does not change much. Therefore, one may attribute the increase of $B_{A}$ and $\eta_{\max }$ to the increase of $\mathrm{Mn}$ luminescence centers. In the high Mn concentration region above $0.5 \mathrm{wt} \%$, both $B_{A}$ and $\eta_{\max }$ decrease rapidly. Two mechanisms may be responsible for the decrease of $\eta_{\max }$. One is the decrease of the mean free path of electrons, which is due to the scattering of hot electrons by the Mn centers and/or the decrease of the crystallinity in the $\mathrm{ZnS}: \mathrm{Mn}$ layer. The other is the increase of the nonradiative transition probability of the excited Mn centers. The existence of a nonradiative transition process is plausible, because the decrease in the decay time $\tau$ of the Mn EL emission was observed in our experiments. Both mechanisms are present in the electroluminescence of the $\mathrm{ZnS}: \mathrm{Mn}$ film, but it is not yet clear which mechanism is predominant.

Further, we have investigated the dependences of $B_{A}$, $\eta_{\max }, E_{A}, J_{A}$, and $\tau$ on the $\mathrm{Tb}$ concentration in $\mathrm{ZnS}: \mathrm{TbF}_{3}$ films. Preliminary results show that the dependences of these quantities (except for $\tau$ ) on the Tb concentration are similar to the case of $\mathrm{ZnS}: \mathrm{Mn}$ films. In $\mathrm{ZnS}: \mathrm{TbF}_{3}$ films, $\tau$ increases with the $\mathrm{Tb}$ concentration, which is contrary its behavior in $\mathrm{ZnS}: \mathrm{Mn}$ films. A detailed analysis and comparison of the dependences of $B_{A}, \eta_{\max }, J_{A}, E_{A}$, and $\tau$ upon the $\mathrm{Mn}$ and $\mathrm{Tb}$ concentrations will give us a clearer knowledge of the mechanism of electroluminescence in $\mathrm{ZnS}$ films.

\section{D. $B-V$ hysteresis characteristic}

ZnS:Mn EL devices having a sandwich structure show the $B$ - $V$ hysteresis characteristic when the Mn concentration is above $0.5 \mathrm{wt} \%$. EL devices with a $\mathrm{ZnS}: \mathrm{Mn}$ film thickness of $6000 \AA$ and an Mn concentration of $0.7 \mathrm{wt} \%$ were prepared at the substrate temperature $140{ }^{\circ} \mathrm{C}$ during evaporation. The typical $B-V$ characteristic of the device is shown in the inserted figure in Fig. 7. The hysteresis width $V_{\text {HYS }}$ is defined as the voltage width at about 1-fL brightness level on the hysteresis loop, as shown in the figure. The dependence of-the hysteresis width on the Mn concentration has already been shown in Fig. 6 and its dependence on the film thickness is shown in Fig. 7. As already reported, ${ }^{4,8,10} V_{\text {HYS }}$ increases rapidly just above the threshold Mn concentration of $0.5 \mathrm{wt} \%$ and increases nearly proportionally to the increase of film thicknesses above $2000 \AA$.

Many studies have been reported and several mechanisms have been proposed about the memory effect. ${ }^{3,4,6-8,10,16}$ However, none of them seems to have been successful in giving a consistent and satisfactory explanation about the experimental results of the memory effect, such as the dependences of the hysteresis width on the Mn concentration and the film thickness. Here, we wish to discuss the mechanism of the memory effect, taking into account our experimental results with $\mathrm{ZnS}: \mathrm{TbF}_{3}$ EL devices, since $\mathrm{ZnS}: \mathrm{TbF}_{3}$ devices show no memory effect even if the $\mathrm{Tb}$

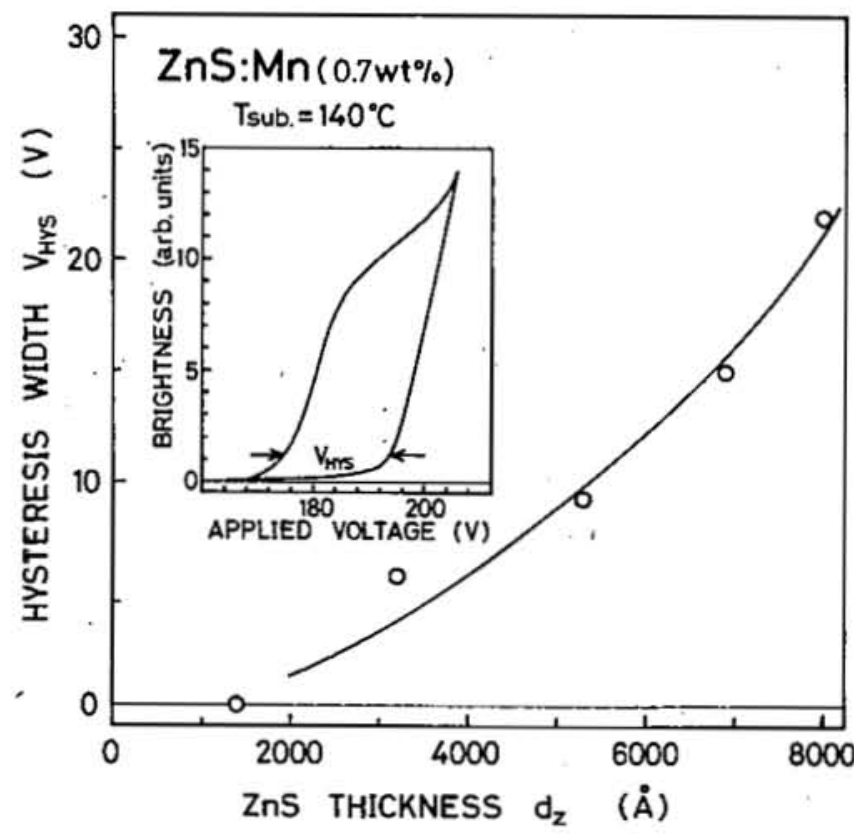

FIG. 7. Dependence of hysteresis width $V_{\text {HYs }}$ on the film thickness of the $\mathrm{ZnS}: \mathrm{Mn}$ active layer of the device. The typical $B$ - $V$ hysteresis characteristic of the device is also shown in the inserted figure. 
concentration exceeds $5 \mathrm{wt} \%$. In the higher concentration region, both $\mathrm{ZnS}: \mathrm{Mn}$ and $\mathrm{ZnS}: \mathrm{TbF}_{3}$ devices show an increase of $E_{A}$ and a decrease of $J_{A}$. However, the hysteresis of the conduction charge is observed only in the $\mathrm{ZnS}: \mathrm{Mn}$ devices. The decrease of $\tau$ is also observed in the $\mathrm{ZnS}: \mathrm{Mn}$ devices, that is, the nonradiative transition probability increases in the concentration region of the memory effect. This indicates that Mn complexes ${ }^{18}$ are formed (as killer centers) in the higher Mn concentration-region. Therefore, the main cause of the hysteresis characteristics observed in the $\mathrm{ZnS}: \mathrm{Mn}$ devices is presumably the electron traps of the Mn complexes, which are formed in the $\mathrm{ZnS}: \mathrm{Mn}$ layer at higher Mn concentrations. However, the memory effect is a very complicated phenomenon, and further detailed study is necessary for a more perfect understanding of its nature.

\section{SUMMARY}

We have investigated systematically the dependences of the electroluminescent characteristics of $\mathrm{ZnS}: \mathrm{Mn}$ EL thinfilm devices upon the device parameters, such as crystallinity, film thickness, and Mn concentration of the $\mathrm{ZnS}: \mathrm{Mn}$ active layer. The results are summarized as follows.

(1) The $\{111\}$ surface of the cubic-ZnS:Mn film is parallel to the substrate at substrate temperatures during evaporation of $80 \sim 160^{\circ} \mathrm{C}$. The substrate temperature affects the crystallinity of the films. The x-ray diffraction intensity $I_{11}^{*}$ increases with the film thickness, and it tends to saturate above $3000 \AA$. The increase of $I_{11}^{*}$, shows that the crystallinity increases with the film thickness.

(2) In the region of film thicknesses below $3000 \AA$, the maximum EL emission efficiency $\eta_{\max }$ increases. However, the average electric field of the $\mathrm{ZnS}: \mathrm{Mn}$ layer decreases with the film thickness. The increase of $\eta_{\max }$ can be explained by the increase of the mean free path $\lambda$, when the crystallinity increases with the film thickness.

(3) With increasing Mn concentration, $\eta_{\max }$ increases and reaches maximum at about $0.45 \mathrm{wt} \%$. With any further increase of the Mn concentration, $\eta_{\max }$ and $\tau$ decrease rapidly. Also, $E_{A}$ increases slowly and $J_{A}$ decreases slowly. These results may be attributed to the decrease of hot electron ener- gy in the ZnS:Mn active layer and the increase of the nonradiative transition probability of the excited Mn emission centers.

(4) The $B-V$ hysteresis characteristic, the so-called memory effect, is observed in devices with Mn concentrations above $0.5 \mathrm{wt} \%$ and film thicknesses above $2000 \AA$. The mechanism of this effect is probably related to the variation of $E_{A}$ and $J_{A}$, and to the formation of $\mathrm{Mn}$-complex centers. However, the mechanism is too complicated for a completely satisfactory understanding.

'T. Inoguchi, M. Takeda, Y. Kakihara, Y. Nakata, and M. Yoshida, Digest 1974 SID International Symposium (Society for Information Display, Los Angeles, 1975), p. 84.

${ }^{2}$ Y. Yamauchi, M. Takeda, Y. Kakihara, M. Yoshida, J. Kawaguchi, H. Kishishita, Y. Nakata, T. Inoguchi, and S. Mito, Digest 1974 Int. Elec. Device Mtg. (IEEE, New York, 1974), p. 352.

${ }^{3}$ M. Takeda, Y. Kakihara, M. Yoshida, Y. Nakata, M. Kawaguchi, H. Kishishita, Y. Yamauchi, T. Inoguchi, and S. Mito, J. Jpn. Soc. Appl. Phys. 44 (Suppl.), 103 (1975).

${ }^{4} \mathrm{M}$. Yoshida, Y. Kakihara, T. Yamashita, K. Taniguchi, and T. Inoguchi, Jpn. J. Appl. Phys. 17 (Suppl. 17-1), 127 (1978).

s. Tanaka, H. Kobayashi, H. Sasakura, and Y. Hamakawa, J. Appl. Phys. 47, 5391 (1976).

'V. Marrello, W. Rühle, and A. Onton, Appl. Phys. Lett. 31, 452 (1977).

${ }^{7}$ W. Rühle, V.Marrello, and A. Onton; J. Lumin. 18/19, 729 (1979).

"V. Marrello and A. Onton, IEEE Trans. Electron Devices ED-27, 1767 (1980).

'W. E. Howard, IEEE Trans. Electron Devices ED-24, 903 (1977).

${ }^{10} \mathrm{~J}$. M. Hurd and C. N. King, J. Electron. Mater. 8, 879 (1979).

''J. Benoit, P. Benaiioul, R. Parrot, and J. Mattler, J. Lumin. 18/19, 739 (1979).

${ }^{12}$ V. Marrello and A. Onton, Appl. Phys. Lett. 34, 525 (1979).

${ }^{13}$ V.P. Tanninen, T. Tuomi, M. C. Typpi, R. O. Törnqvist, T. Suntola, J. Antson, A. Pokkala, and S. Lindfors, in (Proceedings of the 8th International Vacuum Congress, Cannes, 1980), p. 401.

${ }^{14}$ Y. S. Chen and D. C. Krupka, J. Appl. Phys. 43, 4089 (1972).

${ }^{15}$ H. Kobayashi, S. Tanaka, H. Sasakura, and Y. Hamakawa, Jpn. J. Appl. Phys. 13, 264 (1974).

${ }^{16} \mathrm{~V}$. Marrello and A. Onton, J. Electrochem. Soc. 127, 2220 (1980).

${ }^{17}$ G. A. Baraff, Phys. Rev. A 133, 26 (1964).

${ }^{18}$ W. Busse, H. E. Gumlich, B. Meissner, and D. Thesis, J. Lumin. 12/13, 693 (1976). 